\section{La situación del aborto en Colombia: entre la ilegalidad y la realidad}

\author{
Current situation with abortion in Colombia: \\ between illegality and reality
}

\section{Ministerio de la Protección Social, Bogotá, Colombia. \\ Correspondencia A. C. G. Vélez Ministerio de la Protección Social. Cra. 5 \# 45-30 (B. 3 apto. 504), Bogotá, Colombia. acgonzalez@cable.net.co}

\begin{abstract}
This article discusses the illegality of abortion in Colombia, situating this country within the $0.4 \%$ of the world population where abortion is completely banned. Absolute criminalization of abortion turns it into a public health matter and produces social inequality. The Colombian legislation has always disregarded women as individuals and as persons in full possession of their legal rights. In contrast to a comprehensive conceptualization of sexual and reproductive rights, the various abortion bills merely refer either to "morally unacceptable" situations such as pregnancy resulting from rape or to therapeutic motives. Contradictions between illegality and reality give rise to a public discourse that features rejection of abortion practices, in keeping with the prevailing stance of the ecclesiastic hierarchy, while in practice, and at the private level, people resort to voluntary interruption of pregnancy under conditions of safety and confidentiality, at least for women from the higher socioeconomic strata. This situation not only causes social inequality but also reflects how laws lose meaning and create the collective impression of being useless or unnecessary, thus undermining the state's governing role.
\end{abstract}

Criminal Abortion; Social Inequity; Legislation; Abortion

\section{La situación del aborto en Colombia dentro del contexto latinoamericano}

Colombia es un país lleno de contradicciones. La presencia de una población mayoritariamente devota a la iglesia católica, contrasta con la situación de violencia. Por otra parte, mientras el aborto está penalizado en todas las circunstancias, las estimaciones más conservadoras hablan de 200.000 abortos al año y otras llegan a afirmar que en Colombia suceden alrededor de 400.000 abortos inducidos anualmente 1 . Así es, puesto que las leyes colombianas han permanecido estáticas, penalizando el aborto en todas las circunstancias desde 1936. Esta situación ubica a las mujeres en Colombia, dentro del $0,4 \%$ de la población mundial que vive en países donde el aborto está totalmente prohibido, mientras que el restante $99,6 \%$ de la población se encuentra en países donde el aborto está permitido, al menos para proteger la vida de la mujer.

Con Colombia sólo dos países más en América Latina comparten ese deshonroso lugar prohibiendo el aborto en todos los casos: Chile y El Salvador. El gobierno colombiano y los y las legisladoras colombianas han hecho caso omiso de las múltiples convenciones y tratados internacionales en los que se le ha recomendado a Colombia revisar la penalización absoluta del aborto. A esta situación de ilegalidad, se suma la realidad del aborto como un problema 
de salud pública que durante más de una década ha ocupado entre la segunda y tercera causa de muerte materna en el país. Según estadísticas del Departamento Administrativo Nacional de Estadística (DANE), los embarazos que terminan en aborto corresponden al $16,0 \%$ de las causas de muerte materna 2 . El estudio La Incidencia del Aborto Inducido en Colombia muestra que el $22,9 \%$ de todas las mujeres encuestadas entre 15 y 55 años declaró haber tenido por lo menos una experiencia de aborto inducido y afirma que aunque el riesgo de aborto es alto para todos los grupos de edad "una joven de menos de 19 años embarazada tiene el doble de riesgo de aborto que una mujer mayor de 40 años: casi la mitad de estas jóvenes menores de 19 años embarazadas ha tenido una experiencia de aborto" 3 (p. 50).

Pese a la situación de salud pública y a la ilegalidad que caracteriza a Colombia con respecto a otros países del mundo, el único esbozo de cambio que se ha visto en el país vino con la última reforma al Código Penal en 2000. En este Código, a renglón seguido de la ya conocida penalización para todas las circunstancias, aparece un parágrafo que para algunos ha sido considerado una ventana hacia el camino de la despenalización parcial y para otros una figura retórica que no significa avance alguno en la legislación, ni en el reconocimiento de los derechos de las mujeres. En los delitos contra la vida y la integridad personal, el aborto está penalizado considerando circunstancias de atenuación punitiva cuando el embarazo sea resultado de una conducta constitutiva de acceso carnal o acto sexual sin consentimiento, abusivo, de inseminación artificial o transferencia de óvulo fecundado no consentidas. "Parágrafo: en los eventos del inciso anterior, cuando se realice el aborto en extraordinarias condiciones anormales de motivación, el funcionario judicial podrá prescindir de la pena cuando ella no resulte necesaria en el caso concreto" 4 . Sin embargo, vale la pena anotar que en esta misma reforma al Código Penal se tipificaron como delito, por primera vez en el país, las llamadas "lesiones al feto" que una vez más exponen el menor valor relativo que se da a la vida de las mujeres, en relación con el valor que se otorga a la vida fetal, al situar a la mujer en condición delictiva, por ejemplo, por el solo hecho de padecer una enfermedad que pueda ser transmitida al feto durante el embarazo o el parto.

\section{Intentos de cambio en la legislación sobre aborto}

Después de que en 1973 el Senador del Movimiento Cristiano, Fernando Mendoza Ardila, presentara al Congreso un proyecto de ley que tenía como propósito dar entre 16 y 30 años de prisión a "la madre que quite la vida a su hijo futuro, fruto de acceso carnal violento o de inseminación artificial no consentida", se han discutido en el país en estas dos décadas un número de aproximadamente ocho proyectos de ley y se han presentado cuatro demandas de inconstitucionalidad relacionadas con el tema del aborto y dos intentos de reforma al Código Penal. Dejando de lado el proyecto del año 1973, en el que de manera franca y abierta se propone una legislación en la que con la penalización absoluta del aborto se criminalizan, como menciona la Corte Constitucional Colombiana, "conductas no exigibles de una persona, entre ellas la de continuar un embarazo que es producto de una violación. Condiciones, en las que es difícil entender o aceptar el hecho de que la mujer que aborta sea considerada una delincuente" 5 (p. 16), los otros proyectos presentados tienen elementos que ofrecen al análisis de esta situación varias reflexiones.

La mayoría de estos proyectos, presentados en su momento por senadores y senadoras liberales, si bien buscan avances para despenalizar el aborto en algunas circunstancias comunes a casi todos ellos, como el peligro para la vida o la salud de la mujer, la violación, y las malformaciones del feto, entre otras, incluyen en sus requerimientos la autorización expresa del marido, dejando de lado la autonomía reproductiva de las mujeres que debería primar en estas circunstancias. A esto se suma el requerimiento de comprobar la violación por parte de un juez, confirmando cómo en las decisiones reproductivas de las mujeres y en el dominio del territorio de su propio cuerpo, la legislación siempre ha desconocido el respeto a las mujeres como personas y sujetos plenos de derechos. Sólo dos de estos proyectos avanzan un poco al proponer que se permita el aborto también en caso de que existan condiciones socioeconómicas que hagan imposible la gestación para las mujeres, cuando estas personalmente así lo consideren, y uno de ellos explica claramente en su argumentación que se trata de proteger la vida de la mujer y no la del feto, que no es un sujeto activo de derechos. En términos generales estos proyectos no parten 
de una concepción integral sobre los derechos sexuales y reproductivos y se reducen, por lo general, a los argumentos terapéuticos o a las situaciones "moralmente inaceptables", como el embarazo por violación.

De acuerdo a estos proyectos podemos deducir que aproximadamente cada diez años, a partir de los 70, el país ha venido discutiendo el tema del aborto en el Legislativo, proponiendo la despenalización por distintas causas y dejando luego períodos de silencio social y político sobre el tema. Como bien resume Mara Viveros en el artículo El Aborto en Colombia: Veinte Años de Debate en la Prensa 6, podríamos decir que el primer proyecto presentado es resultado de las intensas discusiones sobre el crecimiento demográfico en el mundo y que a este le sigue un intenso período de debates ocasionado por un segundo proyecto de ley que proponía despenalizar en casos de violación, para salvar la vida de la madre y por malformaciones fetales 6 . Según Viveros, en esta época se generaron "el mayor número de artículos que se hayan escrito sobre el tema en los diarios colombianos durante los últimos veinte años" 6 (p. 190). Participaron de este debate tanto los políticos como los juristas, los profesionales médicos, la iglesia y algunas mujeres. A este período le sigue un momento de discusiones desatadas por un proyecto de ley presentado por un senador liberal entre cuyos argumentos más provocadores está el de “...la inoperancia real e inutilidad de la ley al respecto y segundo lugar, la disociación cada vez mayor entre el derecho nacional y la moral social vigente" 6 (p. 200).

\section{Las contradicciones entre la ilegalidad y la realidad}

¿Es la ilegalidad un instrumento

de la doble moral que permite la tolerancia evitando el debate público?

Pese a la ilegalidad absoluta del aborto en Colombia, al menos en algunas de las grandes ciudades del país las mujeres tienen acceso a servicios seguros y de calidad, lo que ha determinado que el aborto haya pasado de ser la primera causa de mortalidad materna en la década de los setenta a ser la tercera causa en los primeros años del siglo XXI. Nuestra hipótesis es entonces que la condición de ilegalidad permite mantener una posición pública y discursiva de rechazo frente a la práctica del aborto, acorde con la posición dominante de la jerarquía eclesiástica, mientras que en la práctica y en el nivel de lo privado se recurre a la interrupción voluntaria de la gestación en condiciones de seguridad y confianza, al menos entre las mujeres de los estratos socioeconómicos más altos. Esta situación paraliza entonces el debate, lo hace "innecesario" y promueve la coexistencia de leyes totalmente restrictivas con prácticas permisivas.

A esta situación se suma hoy el acceso cada vez más generalizado al aborto con medicamentos que hace aun más privada, más segura y más asequible a grupos más amplios de mujeres, la decisión de interrumpir un embarazo. Esto lleva a que se vuelvan invisibles una porción importante de los embarazos no deseados y de los abortos, tranquilizando aún más las conciencias de la doble moral. Paradójicamente entonces, un avance en la técnica que facilita y simplifica el acceso de las mujeres al aborto seguro se convierte en arma de doble filo en la medida que silencia aun más la posibilidad de la discusión y el debate social para la legalización franca y abierta. Esta descripción no desconoce, sin embargo, la presencia aún abundante en el país de una oferta de servicios de aborto que se prestan de manera insegura y sin calidad, poniendo en riesgo la vida y la salud de las mujeres, y que existen solamente gracias a la condición de ilegalidad y clandestinidad del tema en el país. Esta oferta de servicios sólo agranda la brecha de inequidad a la que nos referiremos más adelante.

¿Es la penalización del aborto un instrumento de control o de inequidad social?

Retomando el análisis de las estadísticas de los abortos que suceden en Colombia cada año, podemos concluir que este hecho confirma de manera casi permanente un debilitamiento de la norma y de su función de control. Una ley que se pasa por alto por qué para las ciudadanas son más importantes las consideraciones personales y el balance individual que hace cada una sola o con su pareja, frente a las circunstancias particulares de su momento en la vida, va perdiendo sentido y va creando en el imaginario colectivo la impresión de que las leyes no sirven, ni son necesarias y de esa manera se va debilitando el Estado en su función normativa 6 .

De esa manera la penalización pierde su función de control para convertirse en un instrumento de inequidad social. Se ha demostrado que los efectos más adversos de la clandestinidad los viven las mujeres más vulnerables: aquellas que pertenecen a los sectores socioeconómicos más bajos y las más jóvenes. Son es- 
tas quienes en últimas se ven sometidas a prácticas inseguras, indignas y deshumanizadas.

¿Podrá desaparecer el aborto

de las alternativas contempladas

por las mujeres frente a un embarazo?

Mucho se ha trabajado en identificar los factores que protegen a las mujeres de la ocurrencia de embarazos no deseados y de abortos, concluyendo que el acceso generalizado a la educación y a los métodos anticonceptivos son quizás los factores más importantes para evitar la ocurrencia de estos hechos. Al analizar el caso colombiano a la luz de esos indicadores encontramos que $82,0 \%$ de las mujeres están matriculadas en la educación secundaria y el $76,9 \%$ de las mujeres de 15 a 49 años en unión usan métodos anticonceptivos 7. A esto se suma la existencia de una serie de normas y guías técnicas y una política nacional de salud sexual y reproductiva, que contiene entre sus prioridades la reducción de embarazos no deseados.

Surge entonces la pregunta sobre la razón para que en Colombia más de la mitad de los embarazos sean no deseados y en el país la tasa de abortos (32 abortos por cada 1.000 mujeres entre 15 y 49 años para 1991 y el número de abortos realizados anualmente, sigan siendo persistentes 8 . Comencemos por decir que el uso de métodos anticonceptivos es irregular, en ocasiones inadecuado y discontinuo, lo que conlleva a fallos anticonceptivos frecuentes. Por otra parte, la proporción de relaciones sexuales no consentidas que suceden por cuenta de la violencia intrafamiliar, el abuso y la violencia sexual en contra de las mujeres, hacen muy difícil para algunas de ellas prever un encuentro sexual y por lo tanto protegerse contra el embarazo, al igual que es difícil para muchas negociar el uso de métodos especialmente de protección como el condón.

De otro lado, una cultura que por una parte sobrevalora la maternidad y estimula la sexualidad, pero que por la otra condena y estigmatiza el embarazo ocurrido por fuera de lo "aceptable socialmente", es una cultura que fuerza indirectamente la ocurrencia de abortos. Por lo tanto, basta con decir que por las razones expuestas, entre otras, la prevención no es suficiente y que por lo tanto una proporción mayor o menor de mujeres siempre tendrá que recurrir al aborto para espaciar los nacimientos, para limitar el número de hijos o para ejercer los derechos sobre su propio cuerpo: evitando los embarazos que resultan de violación, salvando su propia vida cuando está en riesgo por la gestación, negándose a ser un "ataúd ambu- lante" de fetos con malformaciones incompatibles con la vida humana o simplemente para tener un hijo en condiciones dignas, tanto de salud mental como de estabilidad socioeconómica 9 .

El caso de Holanda donde la interrupción voluntaria del embarazo no está penalizada y es gratuita, y los anticonceptivos son ampliamente asequibles, y la tasa de aborto es la más baja del mundo, pero aun así está presente, es un claro indicativo de lo aquí enunciado 10. En este escenario el papel del Estado más que el de prohibir e intentar forzar respuestas distintas, debería ser el de tomar medidas para enfrentar el impacto que sobre la salud pública tienen la morbi-mortalidad por aborto, corrigiendo las condiciones de inequidad y desigualdad.

\section{Análisis y conclusiones}

El único camino posible para evitar las muertes por abortos que se practican clandestinamente en forma insegura amparados en la situación de ilegalidad, para evitar la inequidad que se deriva de esta misma ilegalidad y para recobrar el sentido de la norma y propender el ejercicio pleno de la autonomía reproductiva de las mujeres y las condiciones para ejercer sus derechos sexuales y reproductivos, es buscar la legalización del aborto en forma definitiva. Legalización que debe acompañarte de un debate social y público que sea amplio y responsable sobre el tema. Debate que deben asumir sin demora los distintos poderes que tienen en sus manos las decisiones legislativas y políticas del país, y también la sociedad organizada.

Entre los argumentos que tenemos para esta defensa cuatro son claves y resultan de lo expuesto anteriormente: primero que existen condiciones legales nacionales e internacionales que lo sustentan y cuya aplicación se hace necesaria para recobrar el sentido de la norma. La Constitución Nacional y los diversos instrumentos internacionales desde la Declaración de los Derechos Humanos hasta el Plan de Acción sobre Población y Desarrollo 11. Segundo que el aborto es un problema de salud pública, que se genera fundamentalmente por la ocurrencia de abortos inseguros que ocurren al amparo de la clandestinidad. La despenalización del aborto resulta en descensos inmediatos en la mortalidad de las mujeres, mientras que cuando el aborto está penalizado, las mujeres se mueren. En Carolina del Norte (Estados Unidos), la mortalidad materna debida al aborto 
inducido se redujo casi un $85,0 \%$, en un período de cinco años, una vez que el aborto fue despenalizado. En cambio en Rumanía la política pronatal del régimen de Ceaucescu resultó en su momento en la tasa más alta de mortalidad materna en toda Europa (aproximadamente 150 muertes por 100.000 nacidos vivos). Se calcula que el $87,0 \%$ de estas muertes resultaron de abortos clandestinos e inseguros. Tercero que la vida de las mujeres tiene que recobrar su valor social y que en situaciones de conflicto

\section{Resumen}

Este artículo describe la ilegalidad del aborto en Colombia cuya población forma parte del 0,4\% mundial que vive en países donde el aborto está totalmente prohibido. La penalización absoluta hace de este un problema de salud pública generador de inequidad social. En las decisiones reproductivas la legislación siempre ha desconocido a las mujeres como personas, y enfrentada a una concepción integral sobre derechos sexuales y reproductivos, los proyectos de ley apenas se refieren a las situaciones "moralmente inaceptables" embarazo por violación - o a razones terapéuticas. Las contradicciones entre la ilegalidad y la realidad permiten mantener un discurso público de rechazo frente al aborto (posición dominante de la jerarquía eclesiástica), mientras que en la práctica, se recurre a la interrupción voluntaria de la gestación en condiciones de seguridad y confianza al menos entre las mujeres de los estratos socioeconómicos más altos. Esto no sólo genera inequidad social, sino que refleja la forma en que las leyes van perdiendo sentido, creando en el imaginario colectivo la impresión de que no sirven ni son necesarias, debilitando el Estado en su función normativa.

Aborto Criminal; Iniquidad Social; Legislación; Aborto moral, como la que supone la práctica del aborto, el papel de un estado laico y democrático es garantizar la toma de decisiones con respeto pleno de las personas y sus derechos sin imponer los principios de una moral religiosa dominante, y cuarto que el Estado no puede permitir al amparo de la ley situaciones que deriven en inequidad e injusticia social como ocurre para las mujeres más pobres y jóvenes en el caso del aborto.

\section{Referencias}

1. The Alan Guttmacher Institute. Sharing responsibility, women society and abortion worldwide. New York: The Alan Guttmacher Institute; 1999.

2. Ministerio de la Protección Social. Plan de choque para la reducción de la mortalidad materna. Bogotá: Ministerio de la Protección Social; 2004.

3. Zamudio L, Rubiano N, Wartenberg L. La incidencia del aborto inducido en Colombia. Bogotá: Centro de Investigaciones sobre Dinámica Social, Universidad Externado de Colombia; 1994.

4. Régimen Penal Colombiano. Código penal ley 599 del 2000. Bogotá: Legis Editores; 2000.

5. Vargas C, Araujo J, Beltrán A, Cepeda M. Aclaración de voto a la sentencia C-647 de 2001. Bogotá: Corte Constitucional de Colombia; 2001.

6. Viveros M. El aborto inducido en Colombia. Bogotá: Centro de Investigaciones sobre Dinámica Social; 1999.

7. PROFAMILIA. Encuesta Nacional de Demografía y Salud. Bogotá: PROFAMILIA; 2000.

8. Editorial. Int Fam Plann Perspect 1997; 23:111.

9. Diniz D,Ribeiro D. Aborto por anomalia fetal. Brasília: Letras Livres; 2003.

10. Organización Mundial de la Salud. Address unsafe abortion. World Health Day: safe motherhood. Geneva: Organización Mundial de la Salud; 1998.

11. Naciones Unidas. Conferencia Internacional sobre Población y Desarrollo. Plan de Acción del Cairo, 1994. New York: Naciones Unidas; 1994.

Recibido el 17/Ene/2005

Aprobado el 31/Ene/2005 